This is the final peer-reviewed accepted manuscript of:

Phys. Chem. Chem. Phys., 2016,18, 2428-2441.

The final published version is available online at :
http://dx.doi.org/10.1039/c5cp05754j

Rights / License:

The terms and conditions for the reuse of this version of the manuscript are specified in the publishing policy. For all terms of use and more information see the publisher's website.

This item was downloaded from IRIS Università di Bologna (https://cris.unibo.it/)

When citing, please refer to the published version. 


\title{
Doping liquid crystals with nanoparticles. A computer simulation of the effects of nanoparticle shape.
}

\author{
Silvia Orlandi, ${ }^{a}$ Erika Benini, ${ }^{a}$ Isabella Miglioli, ${ }^{a}$ Dean Evans, ${ }^{b}$ Victor Reshetnyak, ${ }^{c}$ and \\ Claudio Zannoni ${ }^{* a}$
}

Received Date

Accepted Date

DOI: $10.1039 / x x x x x x x x x x$

www.rsc.org/journalname

\begin{abstract}
We have studied, using Monte Carlo computer simulations, the effects that nanoparticles of similar size and three different shapes (spherical, elongated and discotic) dispersed at different concentrations in a liquid crystal (LC), have on the transition temperature, order parameter and mobility of the suspension. We have modelled the nanoparticles as berry-like clusters of spherical LennardJones sites and the NP with a Gay-Berne model. We find that the overall phase behaviour is not affected by the addition of small amounts $\left(x_{N}=0.1-0.5 \%\right)$ of nanoparticles, with the lowest perturbation obtained with disc-like nanoparticles at the lowest concentration. We observe a general decrease of the clearing temperature and a reduction in the orientational order with a change in its temperature variation, particularly in the case of the $x_{N}=0.5 \%$ dispersions and with a more pronounced effect when the nanoparticles are spherical.
\end{abstract}

\section{Introduction}

Doping liquid crystals (LCs) with nanoparticles (NPs) provides an important strategy for tuning their properties, since the resulting suspensions may exhibit significantly different and possibly improved features with respect to those of the pure materials, while showing a long term stability essential for industrial applications. Potentially, one of the most important applications is in the field of LC displays (LCD), since doping a nematic LC with small amounts of NPs has been reported to decrease the threshold and switching voltages as well as to reduce the display switching times ${ }^{1-6}$. For these applications an advantage of employing NPs is that optical transparency, essential for displays, can be maintained as scattering is relatively negligible in the visible for these objects of size much smaller than the wavelength.

In a quite different area, various attempts at the bottom up fabrication of metamaterials as self assembled regularly organized suspensions of gold NPs in LCs have been reported ${ }^{7}$. Coating the Au NPs with specific ligands allows NPs to be mixed into other media without segregation, without affecting the LC molecular organization. Sub-wavelength metallic or semiconductor particles can be used to tailor the optical response, achieving very high or very low and negative values of refractive index, permittivity

\footnotetext{
a Dipartimento di Chimica Industriale "Toso Montanari" and INSTM, Università di Bologna, Viale Risorgimento 4, IT-40136 Bologna, Italy; E-mail: claudio.zannoni@unibo.it

${ }^{b}$ Air Force Research Laboratory, Materials and Manufacturing Directorate, WrightPatterson Air Force Base, Ohio 45433, USA. E-mail: dean.evans@us.af.mil

${ }^{c}$ National Taras Shevchenko University of Kyiv,UA-01601 Kyiv, Ukraine. E-mail: victor.reshetnyak@gmail.com
}

and/or permeability ${ }^{8}$.

The modification of LCs properties is, naturally enough, dependent on the type and intrinsic characteristic of the NPs used for doping. Specific features can be introduced, for instance paraand ferromagnetic particles added to nematics are promising candidates for magnetically tunable structures. In an effort to try and understand the behavior of NP - LC composites, a convenient universal observable to monitor is the nematic - isotropic transition temperature $\left(T_{N I}\right)$. In Table 1 we report, without trying to be comprehensive, a few experimental results from literature and even from this small selection we see that the effect on $T_{N I}$ can be very different, depending on the chemical composition, size and shape of the NP, and very difficult to rationalize. Some NP types seem to have a consistent behaviour, e.g. metal NPs such as gold and silver have a tendency to lower the transition temperature ${ }^{9-11}$, even if their surfactant coating may be important in providing exceptions $^{28}$. The addition of very small silica NPs (aerosils) seems instead to have very little effect on the transition temperature, possibly due to the formation of aggregates, which perturb the local order to a lesser extent. ${ }^{20-22}$. Yet another different behaviour is shown by particles somehow chemically similar to aerosils, i.e. clay particles, but with different shape, i.e. platelet-like instead of spherical - like, which display contrasting effects depending on the concentration ${ }^{30}$.

A lowering of $T_{N I}$ and a decrease of $\left\langle P_{2}\right\rangle$ with inorganic NP of $\mathrm{SiO}_{2}$ and $\mathrm{MgO}$, has been obtained by Kobayashi et al. ${ }^{18}$ who argued that the reduction of the effective elastic constant linked to the decreased order is beneficial in lowering the threshold voltage in a twisted-nematic (TN) LCD cell. The NI transition tem- 


\begin{tabular}{|c|c|c|c|c|}
\hline LC system & NP type & NP size $(\mathrm{nm})$ & NP conc & effect on $T_{N I}$ \\
\hline HAT4 $^{9}$ & $\mathrm{Au}$ & 5 & $0.2,0.6,1.2 \mathrm{wt} \%$ & lower $T_{\text {ColI }}$ \\
\hline $6 \mathrm{CHBT}^{10}$ & $\mathrm{Au}$ & $3-5$ & $2 \cdot 10^{-4}, 10^{-3} \mathrm{v} \%$ & lower $T_{N I}$ \\
\hline Polymer $^{11}$ & $\mathrm{Ag}$ & $2-4$ & $1.43,2.97,4.64 \mathrm{wt} \%$ & lower $T_{N I}$ \\
\hline $8 \mathrm{CB}^{12}$ & $\mathrm{BaTiO}_{3}$ & 12 & $0.2-0.4 \mathrm{wt} \%$ & lower $T_{N I}$ \\
\hline $\mathrm{PCPBB}^{13}$ & $\mathrm{Au}$ & 4 & $0.5-5 \mathrm{wt} \%$ & lower $T_{N I}$ \\
\hline $5 \mathrm{CB}^{14}$ & PMMA NPs & 150 & $2-15 \mathrm{v} \%$ & lower $T_{N I}$ \\
\hline $\mathrm{MBBA}^{14}$ & PMMA NPS & 150 & $2-15 \mathrm{v} \%$ & lower $T_{N I}$ \\
\hline $6 \mathrm{CHBT}^{15}$ & $\mathrm{Fe}_{3} \mathrm{O}_{4}$ & 10 & $0.001-0.1 \mathrm{v} \%$ & lower $T_{N I}$ \\
\hline LMW LC $^{16}$ & $\mathrm{CdSe}$ & 4.2 & $0.25-7 \mathrm{v} \%$ & lower $T_{N I}$ \\
\hline $5 \mathrm{CB}^{17}$ & $\mathrm{Au}-\mathrm{SC}_{6} \mathrm{H}_{13}$ & $1.5-2.0$ & $1-10 \mathrm{wt} \%$ & lower $T_{N I}$ \\
\hline $\mathrm{ECB}^{18}$ & $\mathrm{MgO}, \mathrm{SiO}_{2}$ & 10 & $1 \mathrm{wt} \%$ & lower $T_{N I}$ \\
\hline ZLI-4801 ${ }^{19}$ & $\mathrm{Sn}_{2} \mathrm{P}_{2} \mathrm{~S}_{6}$ & 10 & $0.3 \mathrm{wt} \%$ & same $T_{N I}$ \\
\hline $8 \mathrm{CB}^{20}$ & R812 aerosil & 7 & $1-10 \mathrm{wt} \%$ & same $T_{N I}$ \\
\hline $5 \mathrm{CB}^{21}$ & R812 aerosil & 7 & $0.3-3 \mathrm{wt} \%$ & same $T_{N I} \&$ lower $\left\langle P_{2}\right\rangle$ \\
\hline $5 \mathrm{CB}^{22}$ & R812 aerosil & 7 & $1-10 \mathrm{wt} \%$ & same $T_{N I}$ \& lower $\left\langle P_{2}\right\rangle$ \\
\hline $\mathrm{E}^{23}$ & MWNT & $20000-50000$ & $0.1-0.2 \mathrm{wt} \%$ & higher $T_{N I}$ \\
\hline $5 \mathrm{CB}^{24}$ & $\mathrm{BaTiO}_{3}$ & $11-50$ & $0.1-1 \mathrm{wt} \%$ & higher $T_{N I}$ \\
\hline $6 \mathrm{CHBT}^{25}$ & Fe nanorod & $d=20, l=400$ & $5 \cdot 10^{-4} \mathrm{v} \%$ & higher $T_{N I}$ \\
\hline MLC- $6609^{26,27}$ & $\mathrm{BaTiO}_{3}$ & $50-100$ & $0.2 \mathrm{wt} \%$ & higher $T_{N I}$ \\
\hline $5 \mathrm{CB}^{24}$ & $\mathrm{Sn}_{2} \mathrm{P}_{2} \mathrm{~S}_{6}$ & 11 & $0.1-1 \mathrm{wt} \%$ & higher $T_{N I}$ \\
\hline Felix-2900-03 28 & $\mathrm{Au}$ (silanized) & 5 & $0.25-0.75 \mathrm{v} \%$ & higher $T_{N I}$ \\
\hline LC $18523^{29}$ & $\mathrm{Sn}_{2} \mathrm{P}_{2} \mathrm{~S}_{6} / \mathrm{BaTiO}_{3}$ & $5-80$ & $1 \mathrm{wt} \%$ & higher $T_{N I}$ \\
\hline LC $1550^{29}$ & $\mathrm{Sn}_{2} \mathrm{P}_{2} \mathrm{~S}_{6} / \mathrm{BaTiO}_{3}$ & $5-80$ & $1 \mathrm{wt} \%$ & higher $T_{N I}$ \\
\hline $5 \mathrm{CB}^{30}$ & SAP clay & $500-2000$ & $0.1-10 \mathrm{wt} \%$ & non monotonic shift \\
\hline $5 \mathrm{CB}^{31}$ & $\mathrm{Sn}_{2} \mathrm{P}_{2} \mathrm{~S}_{6}$ & 20 & $0.3 \mathrm{v} \%$ & $\begin{array}{c}\text { higher or lower } T_{N I} \\
\text { depending on sample preparation }\end{array}$ \\
\hline
\end{tabular}

Table 1 Some reported effects of different NPs in relation to type, size and concentration on the nematic-isotropic transition temperature $T_{N I}$ and on the orientational order parameter $\left\langle P_{2}\right\rangle$. nCB: 4 n-alkyl 4'-cyanobiphenyl; HAT4: 2,3,6,7,10,11-hexabutyloxytriphenylene; 6CHBT: 4-trans-4-n-hexyl-cyclohexyl -isothiocyanato-benzene; PCPBB : 4-pentylphenyl 2-chloro-4-(4-pentylbenzoyloxy) benzoate; MBBA: n-(4-methoxybenzylidene)-4-butylaniline; PMMA: poly(methyl methacrylate); LMW: LC Low molecular weight LC -

3-hydroxy-4-propionylphenyloxy-4-octyl benzoate; SAP clay: amino-modified poly-iso-butylene (SAP 230 TP) stabilised clay platelets;MWNT: multiwalled carbon nanotubes.

perature is instead reported to increase in some nematics, including both single component cyanobiphenyls and commercial mixtures, when doped with strongly anisotropic NPs including carbon nanotubes ${ }^{23}$ (CNT) and ferromagnetic nanorods ${ }^{25}$. Shape anisotropy should be important here and it might be expected that rod-like particles with high aspect ratio (i.e. very elongated), such as many CNTs should cause an increase of order, even on the basis of classical Onsager theory. ${ }^{32}$ On the other hand, many of these CNTs have sizes in the micro, rather than in the nano- range, and in this case director distortions around the dopant particles acting as defects, lead to an increase of elastic free energy, with the possibility of phenomena, such as phase separations, occurring ${ }^{14,33}$. An increase of $T_{N I}$ has also been observed for various ferroelectric particles, such as $\mathrm{Sn}_{2} \mathrm{P}_{2} \mathrm{~S}_{6}$ or $\mathrm{BaTiO}_{3} 19,26,27,31$ and theoretical models have been proposed to explain the effect. In the first theoretical approach, by Li et al. ${ }^{26}$, a key role was attributed to the strong field generated by the very large dipole moment of these NP. In a second one, by Lopatina and Selinger, ${ }^{34}$ the emphasis is on the orientational order of the NPs induced by the host LC, with a NP order parameter which is predicted to be proportional to the LC order parameter while being independent on the ferroelectric NP concentration. However, there does not seem to be a universal consensus even on the basic fact of the increase of $T_{N I}$ upon addition of ferroelectric NPs. For instance, ferroelectric NPs have also been investigated in LCs, like 8CB, where both the $\mathrm{Sm}_{A} \mathrm{~N}$ and NI transitions resulted in being almost unchanged, or slightly lowered, respectively ${ }^{12}$.

It thus appears that, in spite of the large number of experimental observations, the theoretical understanding of how changing even the simplest generic NP features affects the LC properties is still not available. One of the major difficulties in rationalizing the variety of experiments lies in the large number of variables acting at the same time, ranging from the chemical nature of the different LCs and NPs involved and their (typically present) surfactant coating, but also on NP size, shape, dipoles etc. In addition other factors like polydispersity (in terms of both shape and dimensions), concentration and presence of ions seem to be of considerable importance in the study of the interactions with the nematic host. We notice that, by contrast, the effect of simple non-mesomorphic solutes dissolved in a nematic LC is rather predictable and, generally, that of destabilizing the ordered phase, thus lowering the $T_{N I}$ with the onset of a phase separation in isotropic and ordered phase. ${ }^{35,36}$

To make a progress with understanding and applications of 
these materials, the determination of the extent of the influence of each single element is thus crucial and in this respect modelling and computer simulations can be important, as they allow to treat the various elements one at a time, for instance eliminating spurious effects such as polydispersity. In the present paper we follow this approach to shed light and assess the relative roles of two important factors: NP shape and concentration, on the resulting liquid crystalline behaviour, and we develop first a simple model for NPs of arbitrary shape, building them as suitable rigid assemblies of nanospheres. We consider in particular the case of spherical, rod-like and disc-like NPs, as described in detail in the next section together with the modelling of mesogens as GayBerne ellipsoidal particles. We prepare first a host nematic phase and we characterize it as a function of temperature performing a set of Monte Carlo (MC) computer simulations, as reported in Sec.3. We then perform extensive simulations of suspensions of these NP in the nematic phase at three different concentrations and in Sections 4,5,6 we report our findings for the spherical, rod-like and disc-like NPs.

\section{Mesogen and Nanoparticle Models}

We have chosen to model the mesogens as elongated ellipsoidal particles, and the NPs as clusters ("berries") of spherical objects. More specifically, we have considered three different NP shapes, namely spherical, rod-like and disc-like. One possibility of modelling the NP could be that of adopting a simple object like an ellipsoid, with different aspect ratios. This has been used by Glaser and coworkers in their molecular dynamics work on spherical NPs in nematics, one of the very few dealing with the microscopic simulation of NP in LCs ${ }^{37}$.

$$
\begin{array}{c|c|c|c|c|c|c}
0 & \sigma_{x} & \sigma_{y} & \sigma_{z} & \varepsilon_{x} & \varepsilon_{y} & \varepsilon_{z} \\
1 & 1 & 3 & 1 & 1 & .2
\end{array}
$$

(a)

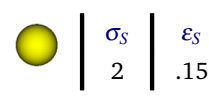

(b)

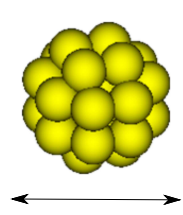

$6.2 \sigma_{0}$

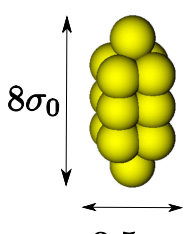

$3.5 \sigma_{0}$

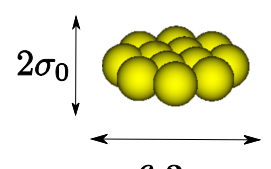

$6.3 \sigma_{0}$ (c)

Fig. 1 Molecular models and parameters for the mesogen (a) and the differently shaped NPs (c). Details of the spherical LJ unit in (b). The distance between the centers of pairs of contiguous and slightly overlapping LJ spheres of radius $1 \sigma_{0}$ is equal to $1.5 \sigma_{0}$

Here we have chosen a different, and more flexible approach, representing NPs of arbitrary shape as tightly-packed clusters of identical Lennard-Jones (LJ) spheres, rigidly connected with each other and slightly overlapping. Apart from this flexibility, we believe that having a multi-site model, rather than a single particle one is definitely more realistic in representing the interactions, localized around the NP surface, between typical low molar mass mesogens and the larger size NPs.
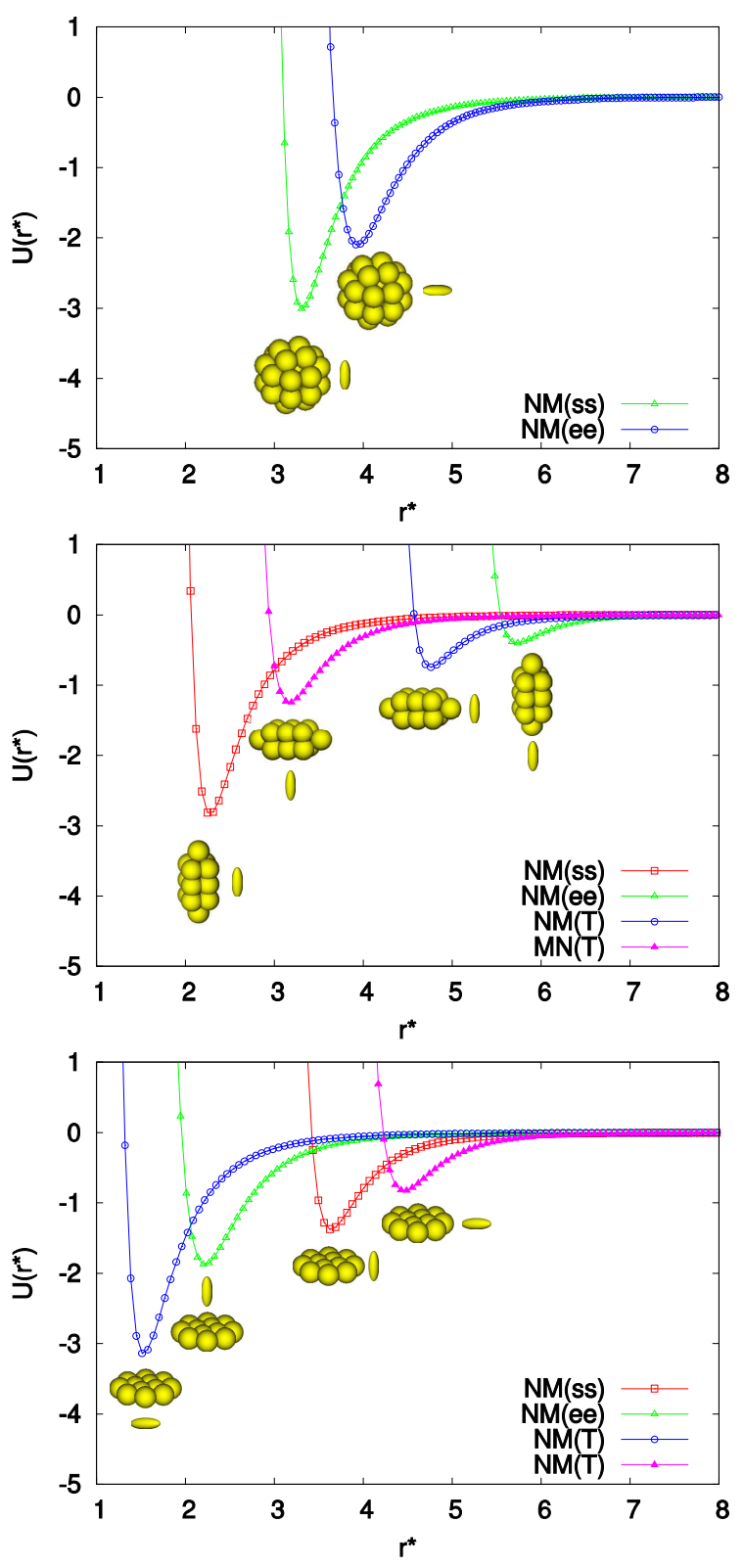

Fig. 2 ss, ee, $T$ energy profiles of heterogeneous NP-mesogen (NM) pair interactions for NPs of spherical, rod-like and disc-like shape. The distance is plotted in $\sigma_{0}$ units whilst the potential in $\varepsilon_{0}$ units.

As for the mesogen, we have employed the well established Gay-Berne (GB) attractive-repulsive potential ${ }^{38,39}$, which is known to be able to generate nematic and smectic LC phases for calamitic molecules and columnar phases for discotics.

The total interaction energy between any pair of species, mesogenic $(\mathrm{M})$ or nanoparticle $(\mathrm{N})$, involves then a summation over all the constituent sites: mesogenic rods or spherical components of the different NPs.

Since the Lennard-Jones potential is just a special case, applicable to spherical objects, of the GB potential between ellipsoids, the interaction between each pair of like or unlike sites, $i$ and 

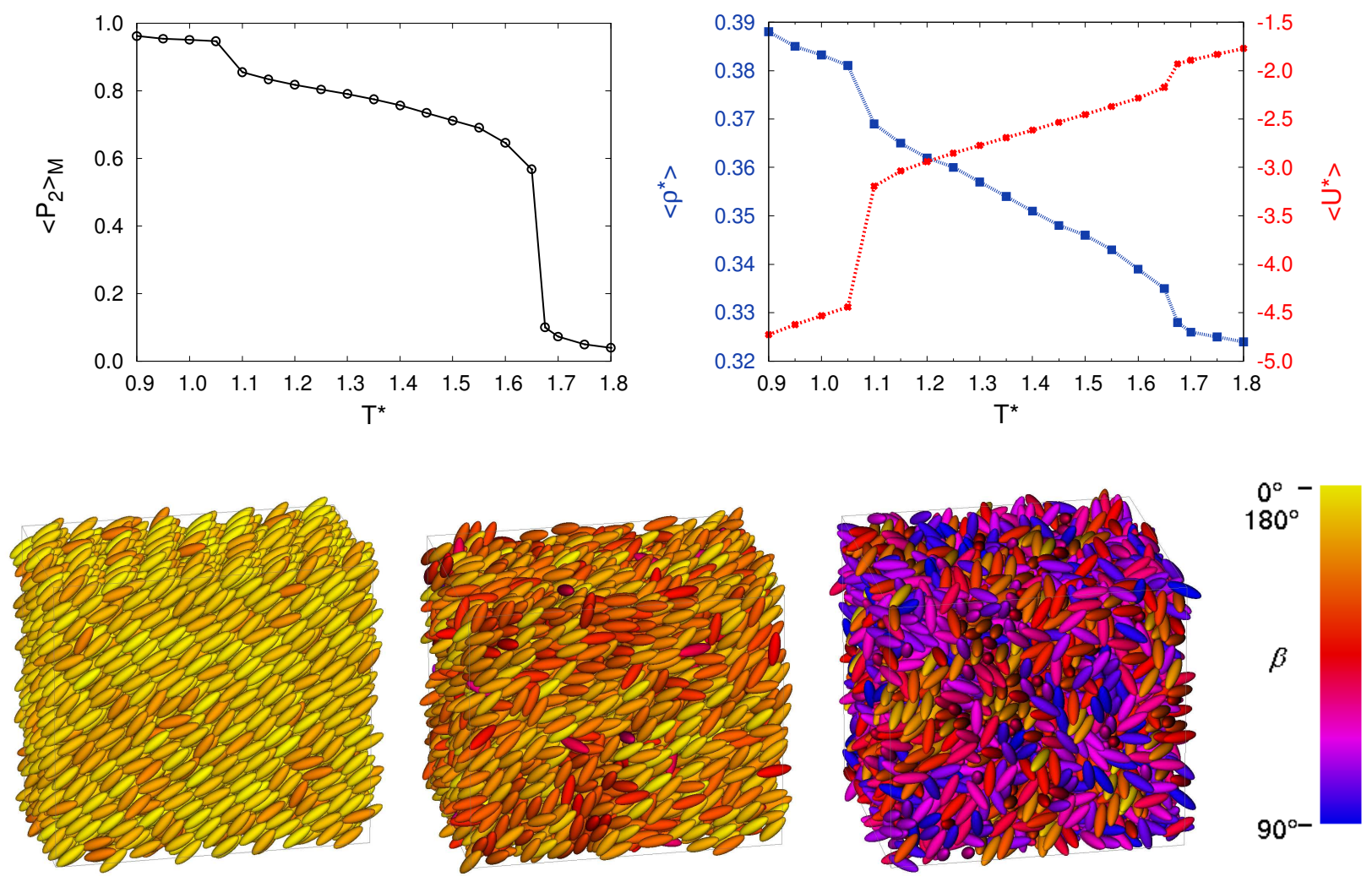

Fig. 3 Average orientational order parameter $\left\langle P_{2}\right\rangle_{M}$ (top left) against dimensionless temperature $T^{*}$ (top left), average number density $\left\langle\rho^{*}\right\rangle=\left\langle N / V^{*}\right\rangle$ (blue) and average dimensionless energy per molecule $\left\langle U^{*}\right\rangle$ (red) vs. $T^{*}$ (top right) for the pure mesogenic system. On the bottom we show three regions corresponding to stable mesophases, i.e. Solid, $T^{*}=1.05$ (left), Nematic, $T^{*}=1.4$ (centre) and Isotropic $T^{*}=1.7$ (right). The particles are coloured according to the angle $\beta$ between their axis and the director, as from the palette shown.

$j$, can be written in any case in terms of the heterogenous GayBerne potential $^{40}$ :

$$
\begin{aligned}
U\left(\omega_{i}, \omega_{j}, \mathbf{r}_{i j}\right) & =4 \varepsilon_{0} e_{i j} \varepsilon\left(\omega_{i}, \omega_{j}, \hat{\mathbf{r}}_{i j}\right)\left[\left(\frac{\sigma_{c}}{r_{i j}-\sigma\left(\omega_{i}, \omega_{j}, \hat{\mathbf{r}}_{i j}\right)+\sigma_{c}}\right)^{12}\right. \\
& \left.-\left(\frac{\sigma_{c}}{r_{i j}-\sigma\left(\omega_{i}, \omega_{j}, \hat{\mathbf{r}}_{i j}\right)+\sigma_{c}}\right)^{6}\right]
\end{aligned}
$$

where $i$ and $j$ could be a mesogen or one of constituent sites of the NPs. Here, the shapes and interactions are described by the contact term $\sigma\left(\omega_{i}, \omega_{j}, \hat{\mathbf{r}}_{i j}\right)$ and by the energy term $\varepsilon\left(\omega_{i}, \omega_{j}, \hat{\mathbf{r}}_{i j}\right)$, that depend on the molecular orientations $\omega_{i}, \omega_{j}$ (not required of course for the case of spherical sites), on the site-site vector $\hat{\mathbf{r}}_{i j}$ and, implicitly, on two parameters $\mu, v$ that allow tuning the shape of the potential well ${ }^{41,42}$. Explicit expressions for $\sigma\left(\omega_{i}, \omega_{j}, \hat{\mathbf{r}}_{i j}\right)$ and $\varepsilon\left(\omega_{i}, \omega_{j}, \hat{\mathbf{r}}_{i j}\right)$ and a more detailed description for the generalized form of the potential allowing also for biaxial molecules may be found, e.g., in reference ${ }^{40}$. Each particle is characterized by its three axes $\sigma_{x}, \sigma_{y}, \sigma_{z}$, its three energy parameters $\varepsilon_{x}, \varepsilon_{y}, \varepsilon_{z}$ (well depths) and the additional well parameter $\sigma_{c}$. The heterogeneous interaction is calculated through the mixing rules adopted by Berardi et al. ${ }^{40}$. To control the strength of the specific interactions, we have also introduced the affinity parameter $e_{i j}$, which scales the strength of site-site pair energy with- out altering the distance dependence. Here all the NP constituent sites have been considered to be identical and both homogeneous terms , $e_{M M}$ and $e_{N N}$, have been set to 1 , while for the mixed ones we have considered $e_{N M}=2.0$.

For the mesogenic host, we have adopted the GB parameterization used originally in references ${ }^{43-45}$ for rod-like LC molecules, namely: shape anisotropy $k=\sigma_{z} / \sigma_{x}=3$, interaction anisotropy $k^{\prime}=\varepsilon_{x} / \varepsilon_{z}=5$, with GB exponential coefficients $\mu=2$ and $v=1$, together with a cutoff distance $R_{c}=4.0 \sigma_{0}$. Here $\sigma_{0}$ and $\varepsilon_{0}$ are used as molecular units of length and energy, which, taking the common mesogen 4-n pentyl, 4' cyano biphenyl (5CB) as our reference elongated molecule, can be approximated to $\sigma_{0} \approx 6 \AA$ and $\varepsilon_{0} \approx 0.4 \mathrm{kcal} / \mathrm{mol}$.

For the three types of NPs (see Fig.1) we have proceeded as follows. The spherical NPs are represented as centrosymmetric berry-like aggregates of 32 identical LJ sites, where each individual LJ site has radius $\sigma_{S}=\sigma_{x}=\sigma_{y}=\sigma_{z}=\sigma_{0}$, so that the overall radius is $\approx 3.1 \sigma_{0}$. Rod-shaped NPs are modelled as clusters of 14 equal LJ spheres with the long and the short axis of the resulting NP of about $8 \sigma_{0}$ and $3.5 \sigma_{0}$. Disc-shaped NPs entail clusters of 12 equal $\mathrm{LJ}$ sites so that the diameter and the thickness of the NP are about $6.3 \sigma_{0}$ and $2 \sigma_{0}$ respectively. In all cases, for the individual LJ unit, we employed the following energetic parametrization: well depth $\varepsilon_{S}=\varepsilon_{x}=\varepsilon_{y}=\varepsilon_{z}=0.15 \varepsilon_{0}$, and model exponents $\mu=2$ and $v=1$.

In Fig. 2 we show the representative pair potential profiles rela- 
tive to the NP-mesogen interaction (NM) for the three NP shapes, obtained summing the pair potential in eq. 1 over all the NP sites. We note that, for spherical NPs, the NM interaction strength (well depth) relative to lateral or side-by-side (ss) configuration is only slightly larger than the terminal or end-to-end (ee) NM interaction. The profiles for rod-shaped NPs evidence that the NM interaction relative to the ss configuration is much larger than both the ee and $T$ interactions (see Fig. 2). Relatively to disc-shaped NPs, the NM interaction relative to one of the two $T$ possible configurations (at $r^{*}=1.5$ ) is much larger than both the face-to-end (at $r^{*}=2.5$ ) and side-by-side $\left(r^{*}=3.5\right)$ interactions.

\section{Pure mesogenic system}

Simulations have been first performed on pure mesogenic systems, that will be considered as a reference for comparing the effect of dispersing the different NPs. Even though the phase diagram of the mesogen potential we employ was well studied in a series of papers by de Miguel and collaborators ${ }^{44,46}$, the interest was mainly focussed to obtaining the general features of the pressure-density phase diagrams, while here we are mainly concerned with the liquid - crystalline order as a function of temperature. This was studied by Emsley et al. ${ }^{47}$ using molecular dynamics at constant density, but for rather small systems (number of molecules $N=256$ ). This size is not adequate for our purpose, particularly as we need to include dopants and, moreover we wish to study our suspensions at constant pressure $P$. We have thus considered systems of $N=4000$ particles in the isobaric-isothermal (NPT) ensemble using 3D periodic boundary conditions, choosing a dimensionless pressure $P^{*}=P \sigma_{0}^{3} / \varepsilon_{0}=8$, while varying the temperatures $T^{*}=k_{B} T / \varepsilon_{0}$ in a range wide enough to observe both isotropic-nematic and nematic-solid transitions of the pure mesogen. The MC updating of particle configurations was performed with a sequence of single molecule positional-orientational random incremental moves. Trial orientations of the particles were generated with the Barker-Watts technique 48 and the maximum angular and positional displacements were adjusted to give an acceptance ratio close to 0.4 . Since we are dealing with NPT simulations, volume-changes were also attempted with a frequency of 1:10; each trial move was performed by randomly selecting one box dimension, assigning it a new length using a random variation and simultaneously linearly rescaling all the molecule centerof-mass positions so as to be consistent with the volume change. For each system, MC equilibrations were run for at least 500,000 cycles (a cycle being a set of $N$ attempted moves) in a cooling sequence starting from isotropic configurations. Production runs, typically also of 500,000 cycles, were then performed to generate the configurations employed for the data analysis. The MC simulations for the systems doped with nanoparticles discussed in the next sections followed the same protocol.

From the equilibrium configurations we computed the average values of the LC molecule orientational order parameter ${ }^{49}$ $\left\langle P_{2}(\cos \beta)\right\rangle_{M}=\left\langle\frac{3}{2} \cos ^{2} \beta-\frac{1}{2}\right\rangle$, where $\beta$ is the angle between mesogen long axis $\mathbf{u}$ and director $\mathbf{n}$. In Fig. 3 we provide some instantaneous snapshots showing three typical configurations in different mesophase regions.

In particular at $T^{*}=1.7$, it is apparent a steep increase in
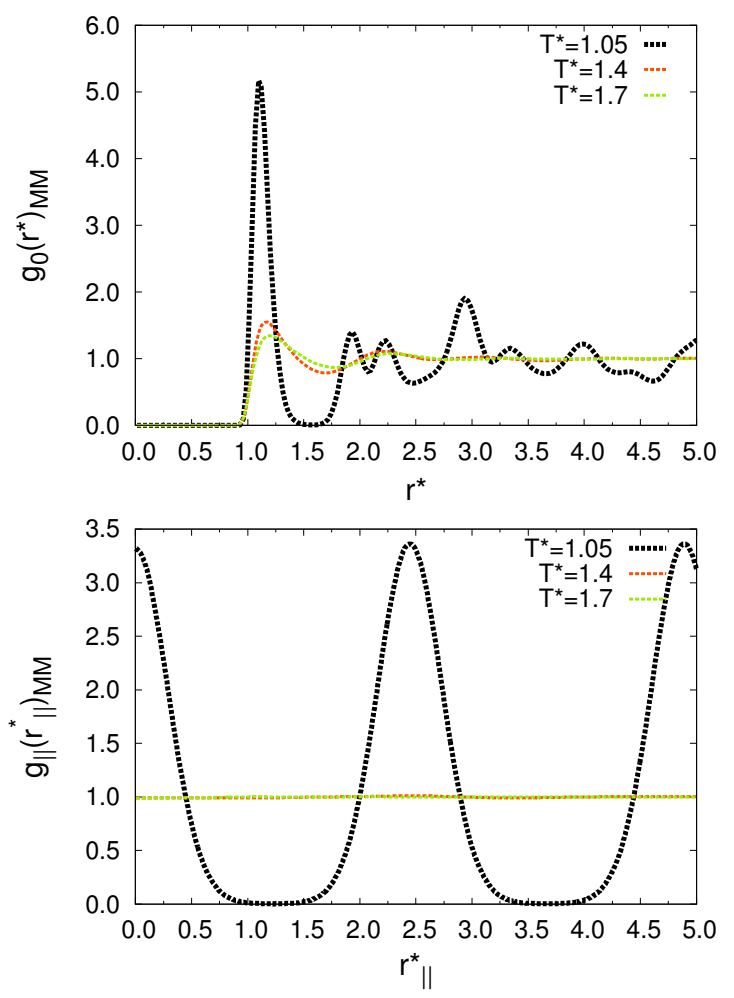

Fig. 4 Radial correlation function (top) and density correlation function (bottom) along the director in solid $\left(T^{*}=1.05\right)$, nematic $\left(T^{*}=1.4\right)$ and isotropic $\left(T^{*}=1.7\right)$ phase for the pure mesogen.

$\left\langle P_{2}\right\rangle_{M}$ corresponding to the first order isotropic-nematic transition, while at $T^{*}=1.1$ a further jump in $\left\langle P_{2}\right\rangle_{M}$ corresponds to the nematic-to-solid (or highly ordered smectic) transition ${ }^{43}$. It is possible to investigate the phase behaviour and identify the transitions also inspecting the average internal energy $\left\langle U^{*}\right\rangle=\left\langle U^{\rangle} / \varepsilon_{0}\right.$ versus $T$, as reported in Fig.3. Two changes of slope are evident, both corresponding to the $T^{*}$ values at which the $\left\langle P_{2}\right\rangle_{M}$ jumps occur. In particular the transition to the low temperature phase is characterized by a large increase of the cohesion energy, which reveals the onset of a more structured phase, more likely to be a solid (S) rather than a smectic $(\mathrm{Sm})^{46,50}$.

In order to evaluate quantitatively the differences caused by the NPs and the deviations from the pure nematic LC behaviour, it is convenient to represent concisely all the variations of $\left\langle P_{2}\right\rangle_{M}$ against temperature, fitting the simulated data points in the nematic phase with the empirical Haller equation: ${ }^{39,51}$

$$
\left\langle P_{2}\right\rangle=\left(1-\left\langle P_{2}\right\rangle_{I}\right)\left(1-\frac{T^{*}}{T_{N I}^{*}}\right)^{\beta}+\left\langle P_{2}\right\rangle_{I}
$$

In eq. $2\left\langle P_{2}\right\rangle_{I}$ is the order parameter in the isotropic phase (which is small but different from zero for a finite size sample) and $T_{N I}$ the nematic-isotropic transition temperature, while the pseudocritical exponent $\beta$ is a fitting parameter that describes the temperature dependence of $\left\langle P_{2}\right\rangle$ in the proximity of the transition. This equation, notwithstanding its empirical nature, has been widely used to successfully approximate experimental ${ }^{52-55}$ and simulated data both for Gay-Berne type models ${ }^{39,47}$ and atom- 

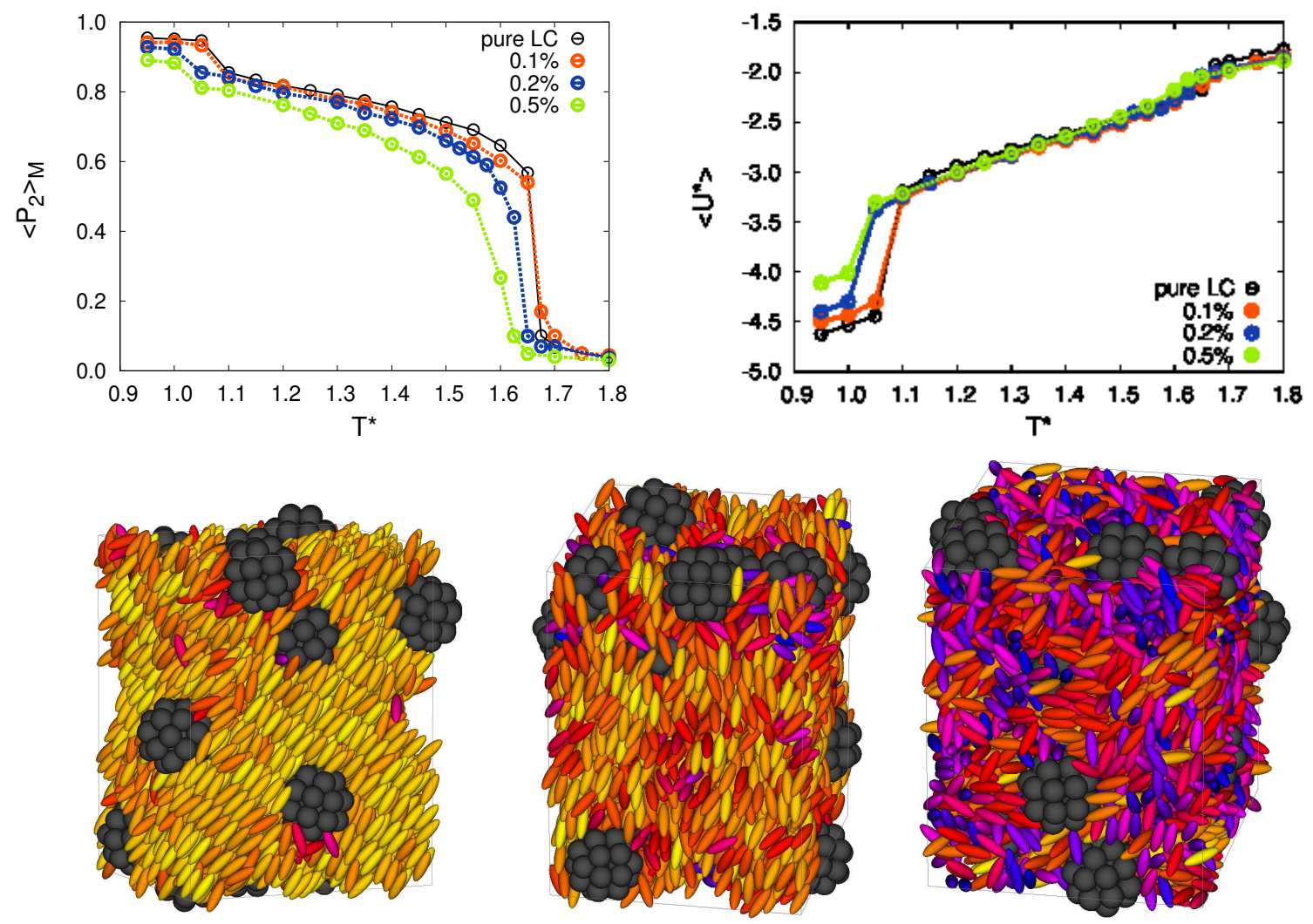

Fig. 5 Average orientational order parameter $\left\langle P_{2}\right\rangle_{M}$ and average dimensionless energy per molecule $\langle U\rangle_{M}$ against the dimensionless temperature $T^{*}$ for three LC/spherical NP dispersions, at increasing mole fraction $x_{N}$; the data for the pure system are reported here for comparison. Snapshots of $\mathrm{x}_{N}=0.5 \%$ dispersions in solid $\left(T^{*}=1.0\right)$, nematic $\left(T^{*}=1.4\right)$ and Isotropic $\left(T^{*}=1.7\right)$ phases: here the multisite NPs are represented in dark gray, while the GB mesogens are colour coded according to their orientation respectively to the phase director (yellow for parallel and blue for orthogonal orientation).

istic ones ${ }^{56}$. For Gay Berne systems, values of $\beta$ were obtained for the present parameterization by Emsley et al. ${ }^{47}$ for their $N=256$ particles systems at fixed densities $\rho^{*}=N / V^{*}=0.30,0.32,0.35$ using molecular dynamics and obtaining $\beta=0.37,0.43,0.45$. Here, operating at constant pressure, and thus with a density that changes with temperature (see Fig.3) we find a rather different value $\beta \approx 0.165$, more similar to the one we found at $\rho^{*}=0.3$ for the Gay-Berne with the parameterization $\mu=1, v=3^{57}$, i.e. $\beta \approx 0.17$. However, we notice that, aside from the very different sample size, a fixed value of $\left\langle P_{2}\right\rangle_{I}$ was used in ref. ${ }^{47}$ irrespective of the actual value resulting from the simulation. It is worth remarking that even from the experimental point of view it is difficult have a unambiguous absolute value. For instance, the values for $5 \mathrm{CB}$, have been experimentally determined by various groups and reported to be $\beta \approx 0.14^{53}, 0.17^{54}, \beta \approx 0.19^{52}$ and $\beta \approx 0.25^{55}$. In any case we are not so much interested here in the absolute values of the pseudo-critical exponent, but rather in using it as a simple indicator of the effects of adding NP to the system when all the other conditions (sample size, model parameters etc. are the same). We shall thus perform a similar Haller fit for all the cases studied and we shall later on examine the differences.

More detailed structural information is provided by the radial distribution function $g_{0}(r)_{M M}$, which estimates the probability of finding a mesogenic molecule at a given distance $r$ from another one chosen as the origin of the coordinate system:

$$
g_{0}(r)_{M M}=\frac{1}{4 \pi r^{2} \rho}\left\langle\delta\left(r-r_{i j}\right)\right\rangle
$$

where $\rho$ is the number density. The pair correlation shows, only in the low temperature case $\left(T^{*}=1.05\right)$, a sharp peak at $r^{*}=1$, corresponding to the side-by-side configuration (see Fig. 4) and also a splitting of the second peak, characteristic of an hexagonal arrangement in the plane, which would suggest the onset of a crystalline/solid phase ${ }^{46}$. In addition, the longitudinal pair correlation $g_{\|}\left(r_{\|}\right)_{M M}$, that expresses the conditional probability of finding two particles separated by a distance $r_{\|}$along the director:

$$
g_{\|}\left(r_{\|}\right)_{M M}=\frac{1}{2 \pi R_{\|} \rho}\left\langle\delta\left(r_{\|}-\mathbf{r}_{i j} \cdot \mathbf{n}\right)\right\rangle,
$$

shows no long-range structure for isotropic and nematic phase, due to the translational disorder of these phases. However, for the low temperature phase, $g_{\|}\left(r_{\|}\right)_{M M}$ shows a strong periodicity with peaks centered at slightly lower values of multiples of the mesogen molecular length, confirming the onset of a well structured layered phase.

\section{LC with embedded spherical-shaped NPs}

We performed simulations of dispersions of $N=N_{M}+N_{N}=4000$ particles in total, with a number of NPs $N_{N}=4,8,20$, corresponding to NP fractional concentration $x_{N}=N_{N} / N=0.1 \%, 0.2 \%, 0.5 \%$ 

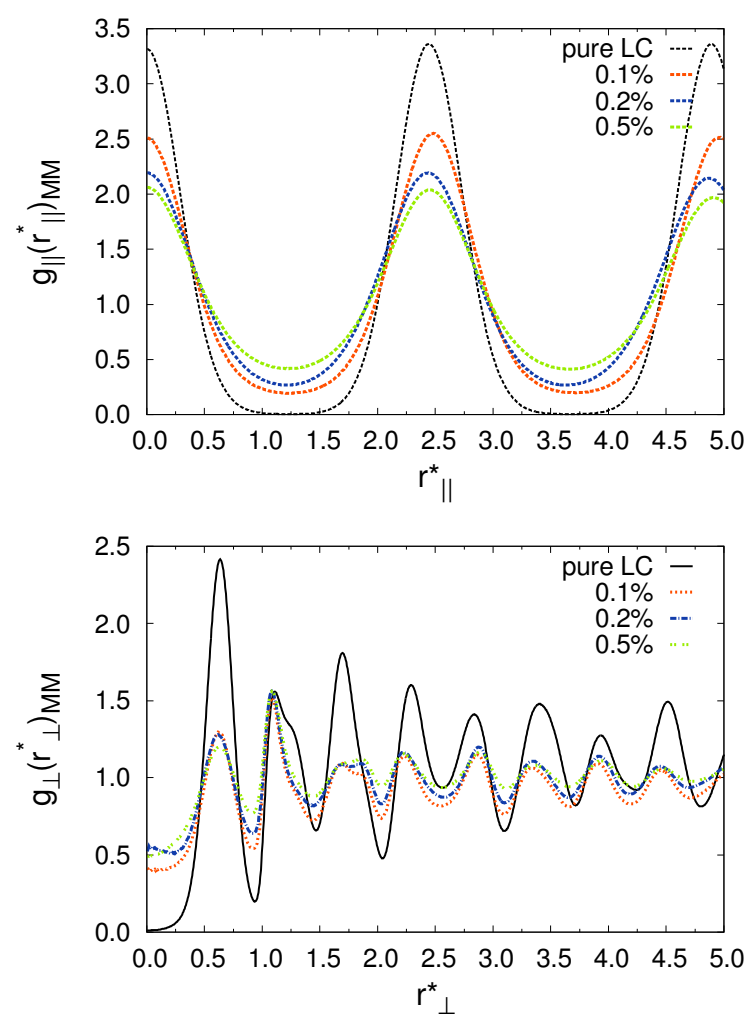

Fig. 6 Longitudinal (top) and transverse (bottom) pair correlation functions referred to mesogenic (M) particles for the LC / spherical -shaped NP dispersions relatively to the highest layered temperature for each $x_{N}$ value (i.e. $T^{*}=1.05$ for $x_{N}=0.1 \%, T^{*}=1.0$ for $x_{N}=0.2 \%$, $0.5 \%)$. The black curves correspond to the behaviour of the pure LC system at $T^{*}=1.05$.

respectively. For each case the simulations were started from an isotropic configuration generated by replacing with the appropriate number of NPs some mesogens of a pure LC sample, previously equilibrated at the same pressure and temperature, and slightly increasing the sample volume to accommodate more easily the NPs. In each case the samples were then equilibrated afresh. The same concentrations were employed for the spherical, rod-like and disc-like NP types that we have considered.

Starting in this section with spherical NPs, we show in Fig. 5 the plot of the temperature dependence of the orientational order parameter of the mesogen, $\left\langle P_{2}\right\rangle_{M}$, compared with that of the pure LC (black line). This exhibits, for the $0.2 \%, 0.5 \%$ cases, a slight uniform shift of both the isotropic-nematic and the nematic-solid transitions towards lower temperatures $\left(\Delta T_{N I}^{*} \approx \Delta T_{S N}^{*} \approx 0.05\right)$; thus the region over which the nematic phase is stable remains unvaried. As the mole fraction is increased, both the first order isotropic-nematic and the nematic-solid become less pronounced and in particular, the energy jump at $T_{S N}^{*}$ reduces. In addition, we see, limitedly to the higher concentration, $x_{N}=0.5 \%$, system, a general decrease of the orientational order of the LC host, and a change in the shape of the $\left\langle P_{2}\right\rangle_{M}$ vs. $T^{*}$ profile. The fit of $\left\langle P_{2}\right\rangle_{M}$ vs. $T^{*}$ to the Haller eq. 2 gives $\beta=0.20,0.22,0.31$ for $x_{N}=0.1$, $0.2,0.5 \%$.

Additional insights into the local structure of the phases can be gained by examining the pair correlation functions for both

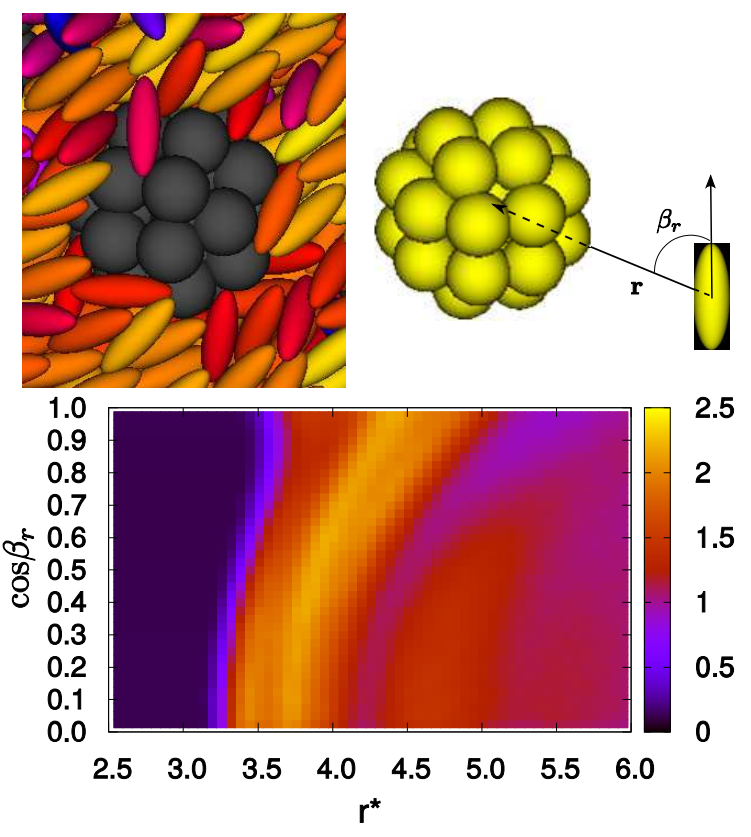

Fig. 7 Close up of a spherical NP environment in a typical instantaneous configuration showing the mesogen anchoring around the NP and a schematic drawing defining the quantities $\mathbf{r}$ and $\beta_{r}$ (top). On the bottom we show a contour map of $g\left(r, \cos \beta_{r}\right)_{N M}$ at $T^{*}=1.4$ for a NP fraction $\mathrm{x}_{N}$ $=0.1 \%$. The palette indicates the intensity of $g\left(r, \cos \beta_{r}\right)_{N M}$, and ranges from 0 to 2.5 .

mesogen-mesogen and NP-mesogen pairs and these have been evaluated. In particular, the structuring of molecular positions in the layered phase can be extracted from $g_{\|}\left(r_{\|}\right)_{M M}$ (eq. 4), and from the transverse pair correlation function, $g_{\perp}\left(r_{\perp}\right)_{M M}$ :

$$
g_{\perp}\left(r_{\perp}\right)_{M M}=\frac{1}{2 \pi R_{\perp} \rho}\left\langle\delta\left(r_{\perp}-\left|\mathbf{r}_{i j} \times \mathbf{n}\right|\right)\right\rangle,
$$

where $R_{\perp}$ is the radius of a cylindrical sampling region. This is shown in Fig. 6 for the highest temperature corresponding to a layered molecular organizations for each $x_{N}$ value, that is $T^{*}$ $=1.05$ for $x_{N}=0.1 \%, T^{*}=1.0$ for $x_{N}=0.2 \%$ and $0.5 \%$. Once again the curves relative to $T^{*}=1.05$ of the pure mesogen are shown for comparison. More specifically, the $g_{\|}\left(r_{\|}\right)$confirms for all $x_{N}$ an undamped sinusoidal modulation with a period similar to the molecular length.

However, we notice that the amplitude of the peaks is always lower than that of the pure mesogen case and also that it decreases for increasing $x_{N}$ : thus the regularity of the layers arrangement turns out to be reduced by increasing the dopant NP fraction. The origin of this smearing out is consistent with the presence of mesogenic molecules between adjacent layers due to the inclusion of the NPs. The complicated structure of $g_{\perp}\left(r_{\perp}\right)_{M M}$ indicates, for any of the NP fractions, a transverse positional correlation that persists across the sample; this is once again consistent with the identification of a solid phase. Note that this function does not correspond to a true two-dimensional distribution since pairs of molecules with considerably different vertical displacement $r_{\|}$, i.e. belonging to different layers, can contribute to $g_{\perp}\left(r_{\perp}\right)_{M M}$. Remarkably, for both the pure mesogen and any $x_{N}$ dispersion, we observe well pronounced peaks at 

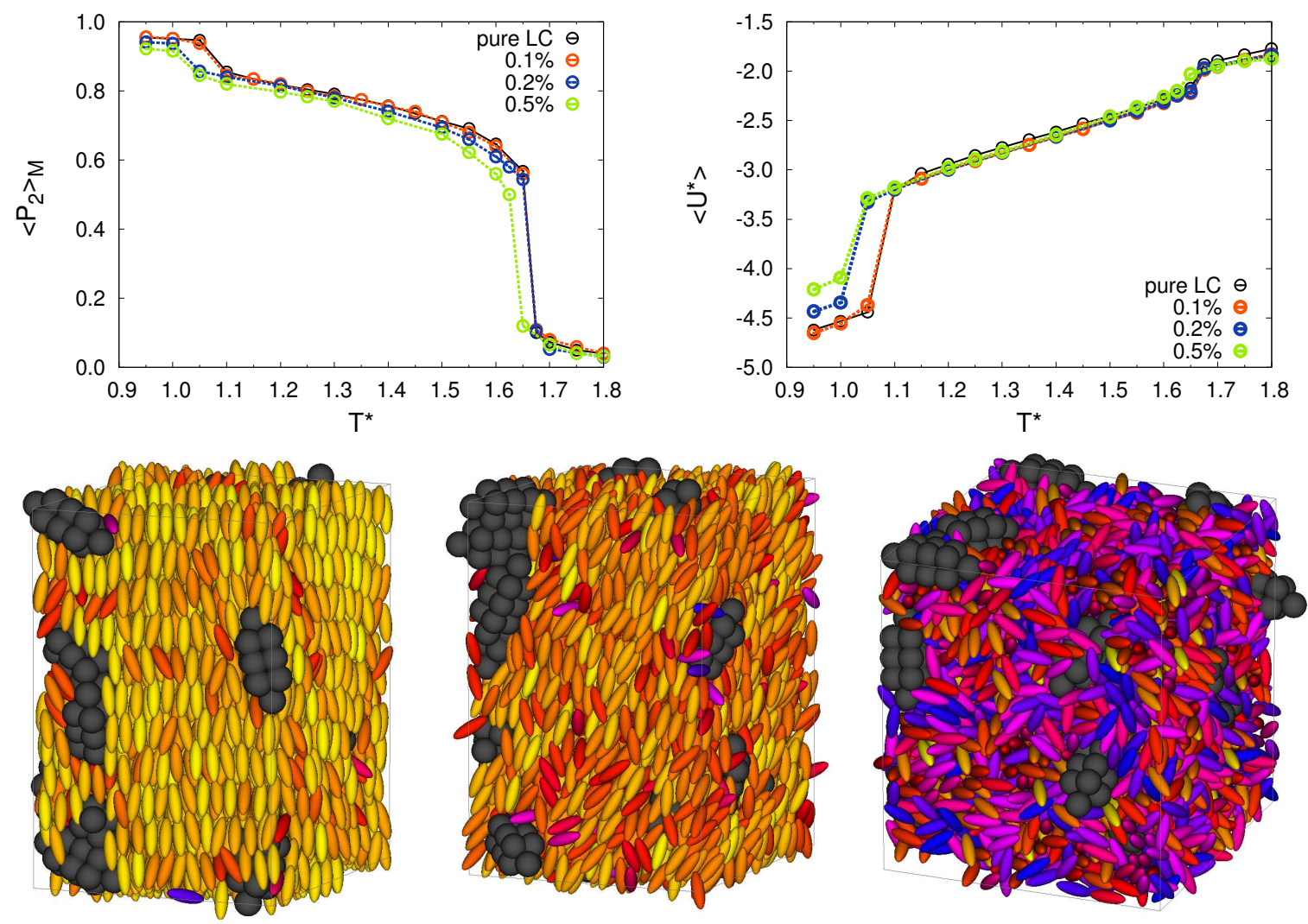

Fig. 9 On the top: average orientational order parameter $\left\langle P_{2}\right\rangle_{M}$ and average dimensionless energy per molecule $\left\langle U^{*}\right\rangle$, against the dimensionless temperature $T^{*}$ for three LC/rod-like NP dispersions at increasing mole fraction $x_{N}$; the data for the pure system are also reported for comparison. On the bottom, from left to right, we show snapshots for $x_{N}=0.5$ at temperatures $T^{*}=1.0,1.4,1.7$.

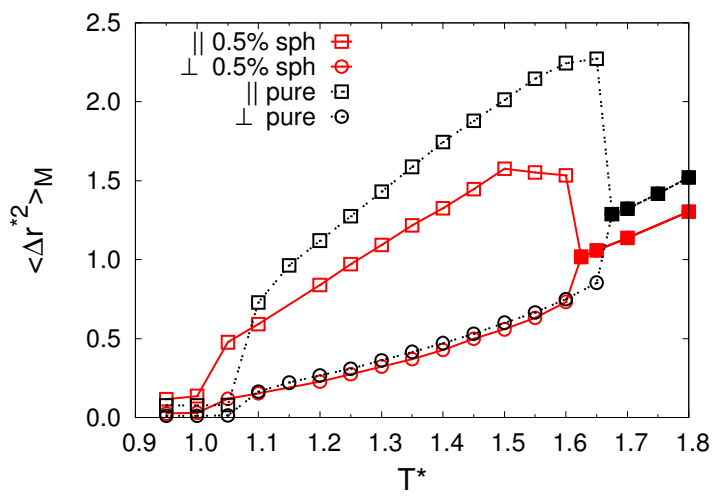

Fig. 8 Temperature dependence of the average MSD components $\left\langle\Delta r_{\|}^{* 2}\right\rangle$ and $\left\langle\Delta r_{\perp}^{* 2}\right\rangle$ measured at $n_{c}=10^{3} \mathrm{MC}$ cycles for the $x_{N}=0.5 \%$

$\mathrm{LC} /$ spherical NP dispersion (in red). The curves in black report the behaviour of the pure LC system.

$r_{\perp}^{*}<1$, which are due to inter-layer correlations and typically appear in the crystal phase. The behaviour of the pure mesogen and the mixtures differs, instead, at very small $r_{\perp}$ : in particular, differently from the pure LC system, the $g_{\perp}\left(r_{\perp}\right)_{M M}$ assumes non zero values at all $x_{N}$, indicating that molecules in adjacent layers do not lie directly one above another. In order to evaluate the NP - mesogen reciprocal arrangement, particularly at the NP surface, we have also examined the anisotropic radial distribution func- tion $^{39} g\left(r, \cos \beta_{r}\right)_{N M}$, which estimates the average arrangement of NP-mesogen pairs as a function of their distance $r$, the modulus of the vector $\mathbf{r}$ connecting their centers of mass and of the angle, $\beta_{r}$, that $\mathbf{r}$ forms with the mesogen long axis (see, e.g., Fig. 7),

$$
g\left(r, \cos \beta_{r}\right)_{N M}=\frac{1}{4 \pi r^{2} \rho}\left\langle\delta\left(r-r_{i j}\right) \delta\left(\cos \beta_{r}-\cos \beta_{i j}\right)\right\rangle
$$

The contour map of this function (Fig. 7), for a $0.1 \%$ sample in the nematic phase, shows that the molecular arrangement at a certain distance from the NP center is not isotropic, but rather varies when the NP- mesogen intermolecular vector is at different angles $\beta_{r}$ with respect to the mesogen molecular axis. The palette on the right side of the graph indicates the distribution function values, which are notably higher along a ring spanning between $r^{*}=3.5, \cos \beta_{r}=0$ (corresponding to a mesogen tangential to the NP surface) and $r^{*}=4.5, \cos \beta_{r}=1$ (corresponding to a mesogen positioned homeotropically on the surface). Overall, the planar mesogens are predominant with respect to the homeotropic ones, indicating the side-by-side intermolecular arrangement as the favourite one, with some exceptions of molecules lying perpendicular, which follow the bulk average alignment and orientation.

We also estimated the relative fluidity of our LCs-NP suspensions by calculating the mean square displacements for the mesogenic species: 


$$
\left\langle\Delta r^{* 2}\left(n_{c}\right)\right\rangle=\left\langle\left|\mathbf{r}^{*}\left(n_{c}\right)-\mathbf{r}^{*}(0)\right|^{2}\right\rangle
$$

where $\mathbf{r}^{*}\left(n_{c}\right)-\mathbf{r}^{*}(0)$ is the displacement vector achieved in $n_{c}$ consecutive MC moves per particle. For an anisotropic particle, the parallel direction is defined as the symmetry axis of the phase (director of the phase), the two independent components being:

$$
\begin{array}{r}
\left\langle\Delta r_{\|}^{* 2}\right\rangle=\left\langle\Delta r_{z}^{* 2}\right\rangle \\
\left\langle\Delta r_{\perp}^{* 2}\right\rangle=\left(\left\langle\Delta r_{x}^{* 2}\right\rangle+\left\langle\Delta r_{y}^{* 2}\right\rangle\right) / 2
\end{array}
$$

so that

$$
\left\langle\Delta r_{i s o}^{*}\right\rangle=\left(\left\langle\Delta r_{\|}^{*}\right\rangle+2\left\langle\Delta r_{\perp}^{*}\right\rangle\right) / 3
$$

Even though in the MC studies we do not have access to real dynamics, we have chosen to perform in our simulations only simple translational and orientational MC moves that mimic the real moves. We thus believe that a comparison of the mean square displacement can allow a comparison of the relative mobility, even if the absolute values of the diffusion coefficients can not be predicted. As expected, a decrease in the mobility when cooling down the systems is evident, with jumps in correspondence of the $T_{N I}^{*}$ and $T_{S N}^{*}$ transitions (see Fig. 8); in addition, in nematic and solid phases, mean square displacements along the director are greater than those perpendicular to it, as in ${ }^{58,59}$. From the comparison with the pure mesogen curves, one can detect two aspects: the perpendicular components $\left\langle\Delta r_{\perp}^{* 2}\right\rangle$ are approximately identical over the whole phase range for both the $0.5 \%$ dispersion and the pure LC; instead, the parallel components $\left\langle\Delta r_{\|}^{* 2}\right\rangle$ estimated for the dispersion are sensibly lower (60\%) than those for the pure mesogenic system in the nematic range, and around twice in the low temperature phase.

\section{LC with embedded rod-shaped NPs}

Turning to the system with embedded rod-shaped NPs, we still observe from the plot of both the orientational order parameter and the internal energy, a $\Delta T_{N I}^{*} \approx 0.05$ shift of NI transition towards lower temperatures, with respect to the pure LC system (black line in Fig. 9) for $\mathrm{x}_{N}=0.5 \%$, together with a $\Delta T_{S N}^{*}=0.05$ shift for $\mathrm{x}_{N}=0.2 \%, 0.5 \%$. Fitting of $\left\langle P_{2}\right\rangle_{M}$ vs. $T^{*}$ to the Haller eq. 2 gives $\beta=0.17,0.18,0.19$ for $x_{N}=0.1,0.2,0.5 \%$. For every temperature and $x_{N}$ value, the NPs tend to have isotropic orientational distributions instead of aligning along the LC phase director and adopt the orientational order of the mesogens.

As before, we analysed the structure of the low temperature phase through the radial correlation functions $g_{\|}\left(r_{\|}\right)$and $g_{\perp}\left(r_{\perp}\right)$, which are reported for the highest temperature corresponding to a layered structure of each $x_{N}$ value, that is $T^{*}=1.05$ for $x_{N}=$ $0.1 \%, T^{*}=1.0$ for $x_{N}=0.2 \%$ and $0.5 \%$ in Fig. 10. For all the $x_{N}$ values, the longitudinal pair correlation $g_{\|}\left(r_{\|}\right)$exhibits (essentially undamped) oscillations similar to those of the pure mesogen. Instead, and differently from the spherical-shaped NPs, only the $x_{N}=0.5 \%$ case exhibits a slight broadening of the peaks intensity: this indicates that the perturbative effect on the layers regularity due to the inclusion of NPs becomes less important if
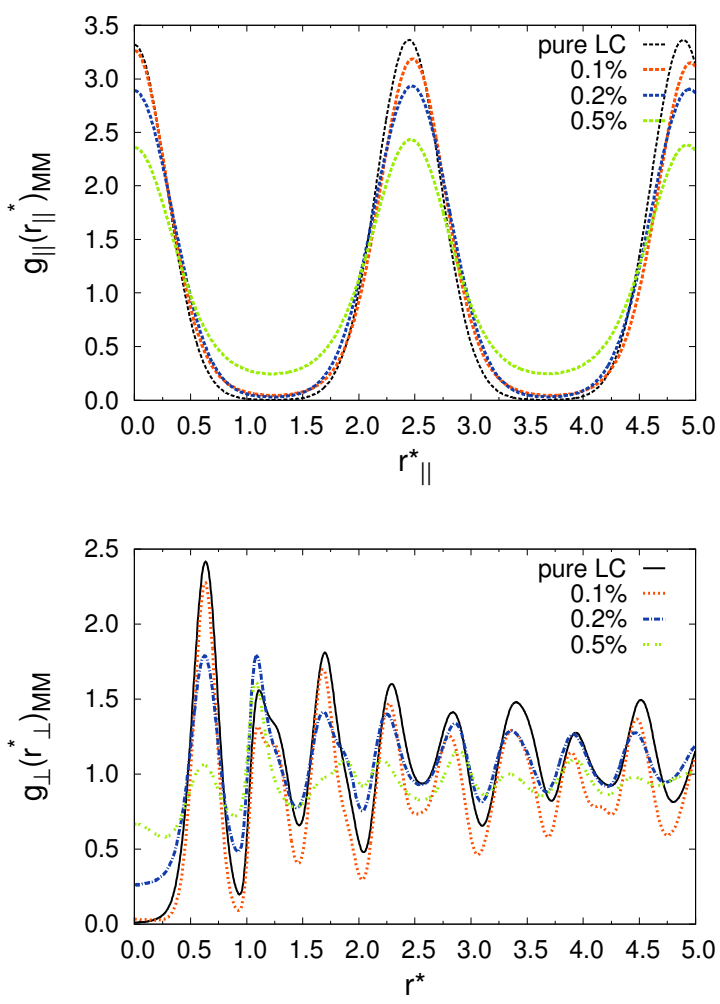

Fig. 10 Longitudinal (top) and transverse (bottom) pair correlation functions referred to M particles for the LC/rod-shaped NP dispersions relatively to the highest layered temperature for each $x_{N}$ value (i.e. $T^{*}$ $=1.05$ for $x_{N}=0.1 \%$ and $T^{*}=1.0$ for $\left.x_{N}=0.2 \%, 0.5 \%\right)$. The curves in black report the behaviour of the pure LC system at $T^{*}=1.05$.

they are rod-shaped rather than spherical. The transversal correlation within the layers, given by $g_{\perp}\left(r_{\perp}\right)_{M M}$, indicates a long range structuring for all $x_{N} \mathrm{x}$. Moreover, limitately to the $x_{N}=0.1 \%$ case, the function falls to zero if $r_{\perp}^{*} \approx 0$, thus suggesting that molecules in adjacent layers are positioned exactly one above another.

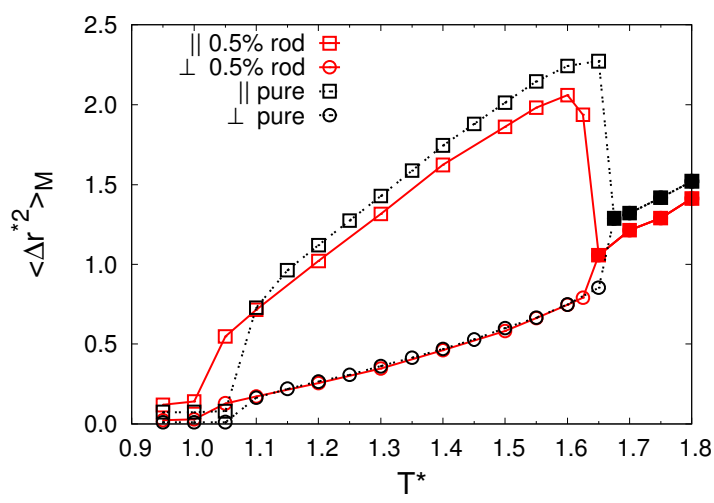

Fig. 11 Temperature dependence of the average MSD components $\left\langle\Delta r_{\|}^{* 2}\right\rangle$ and $\left\langle\Delta r_{\perp}^{* 2}\right\rangle$ measured at $n_{c}=10^{3} \mathrm{MC}$ cycles for the $x_{N}=0.5 \%$ $\mathrm{LC} / \mathrm{rod}$ NP dispersion (in red). The curves in black report the behaviour of the pure LC system.

Looking at the local arrangement between NM pairs, the contour map of the anisotropic radial correlation function for NP- 


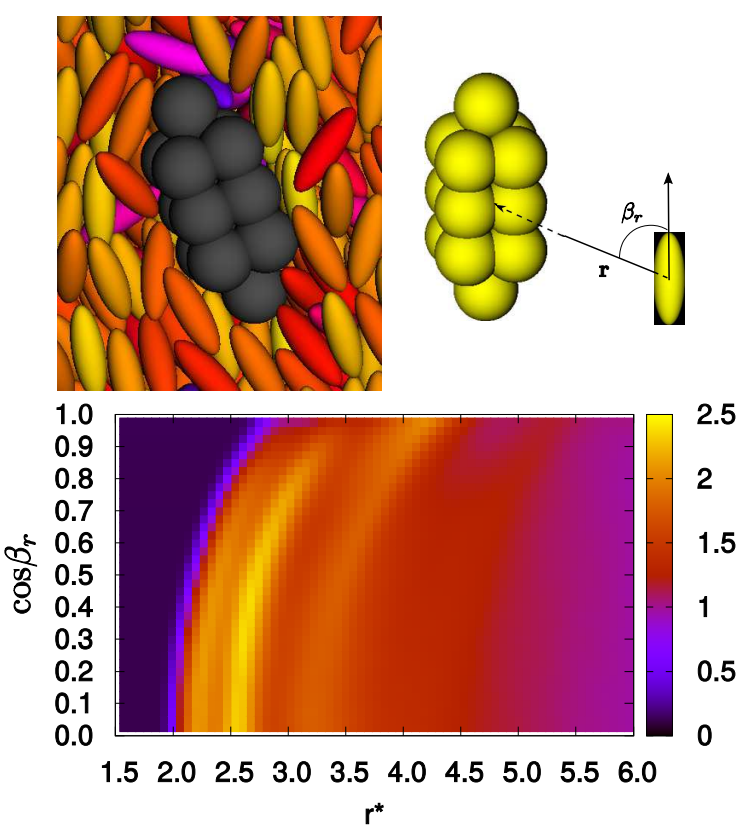

Fig. 12 Longitudinal section of a portion of an instantaneous configuration showing the mesogen anchoring around the rod-shaped $\mathrm{NP}$ and schematical drawing defining the quantities $\mathbf{r}$ and $\beta_{r}$ (top), and contour maps of $g\left(r, \cos \beta_{r}\right)_{N M}$ at $T^{*}=1.4$ for NP fraction $\mathrm{x}_{N}=0.1 \%$ (bottom). The palette on the right indicates the intensity of $g\left(r, \cos \beta_{r}\right)_{N M}$, and ranges from 0 to 2.5 .

mesogen $g\left(r, \cos \beta_{r}\right)_{N M}$ in nematic $\left(T^{*}=1.4\right)$ phase (Fig. 12) displays a set of diffuse arcs; the first, starting around $r^{*} \approx 2.25$, shows a maximum intensity for $\cos \beta_{r}<0.5$ and corresponds to mesogens arranged tangentially on the NP surface: in particular the $\cos \beta_{r}=0$ values corresponds to mesogen-NPs in a side-by-side configuration. Instead, the second, less intense, ring includes for $r^{*} \approx 3.25, \cos \beta_{r}=0, \mathrm{NP}-$ second neighbour mesogens in sideby-side configuration and exhibits the highest values for $r^{*}=4$, $\cos \beta_{r}=1$, corresponding to a mesogen positioned homeotropically at the ends of our rod-shaped NP. Overall, a weak planar anchoring seems to be dominant, even if a portion of homeotropic mesogens is also present, mostly at both ends of the rod. The imperfections in the LC molecule alignment around the NP, and even of the NP surface itself, result in the formation of local orientational defects.

The mesogen mean square displacements against the temperature (plotted for the $x_{N}=0.5 \%$ NP dispersion in Fig.11) show the same, expected, decreasing behaviour already found for spherical NP dispersion. As before, the displacements along the director direction are greater than those perpendicular to it both in nematic phase, where they become comparable to the values of the pure GB system, and in the low temperature phase.

\section{LC with embedded disc-shaped NPs}

The temperature dependence of the LC orientational order parameter of the mesogenic systems doped with disc-like NPs shows the same phase sequence: isotropic-nematic-solid of the pure LC (see Fig. 13), for all the NP concentration values. Unlike the two NP doped systems discussed in the previous sections, only the highest molar fraction values (i.e. $0.5 \%$ ) displays a significant shift in both NI and SN transitions towards lower temperatures, as well as a reduction of the energy jump at $T_{S N}^{*}$, while the two other concentrations closely resemble the pure system trend. This is also shown by the results of the fit of $\left\langle P_{2}\right\rangle_{M}$ vs. $T^{*}$ to the Haller eq. 2 that gives value $\beta=0.17,0.175,0.18$ for $x_{N}=0.1,0.2,0.5 \%$, quite similar to the pure mesogen case.

The local structuring of the low temperature phase relative to the three dispersions at different $x_{N}$, consistent with both the longitudinal and the transverse radial correlation function (see Fig. 14), confirms the weaker disordering effect of embedding disc-like NPs with respect to either spherical or rod-like ones. Here, in fact, even the $x_{N}=0.5 \%$ case shows a negligible broadening of the peaks of $g_{\|}\left(r_{\|}\right)_{M M}$, while the $g_{\perp}\left(r_{\perp}\right)_{M M}$ shows a strong structuring for all values of $x_{N}$, together with an enhanced correlation between adjacent layers for the $0.1 \%$ and $0.2 \%$ cases. We rationalize this behaviour on the basis of the spatial arrangement of the NPs, which can be inferred from an inspection of the snapshots of instantaneous molecular configuration in the low temperature phase (Fig. 13).
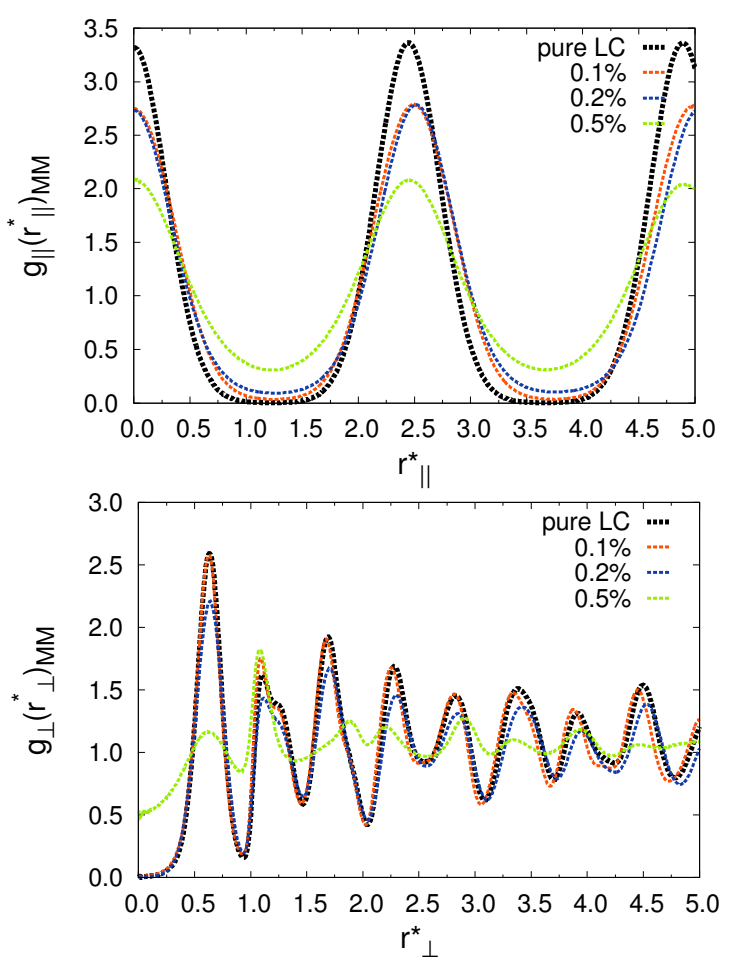

Fig. 14 Longitudinal (top) and transverse (bottom) pair correlation functions referred to M particles for the LC/disc-shaped NP dispersions relatively to the highest layered temperature for each $x_{N}$ value (i.e. $T^{*}$ $=1.05$ for $x_{N}=0.1 \%$ and $0.2 \%$ and $T^{*}=1.0$ for $x_{N}=0.5 \%$ ) (in red). The curves in black report the behaviour of the pure LC system at $T^{*}=1.05$.

Due to their flat shape, NPs can easily accommodate within the ordered structure of the mesogens, with host mesogen molecules aligning parallel to their plane as well as to the LC director. In other words disc-like NP tend to align their axis perpendicularly to the LC phase director and thus the computed $\left\langle P_{2}\right\rangle_{N}$, referred to the LC director, is always negative with values even $<-0.4$ in 

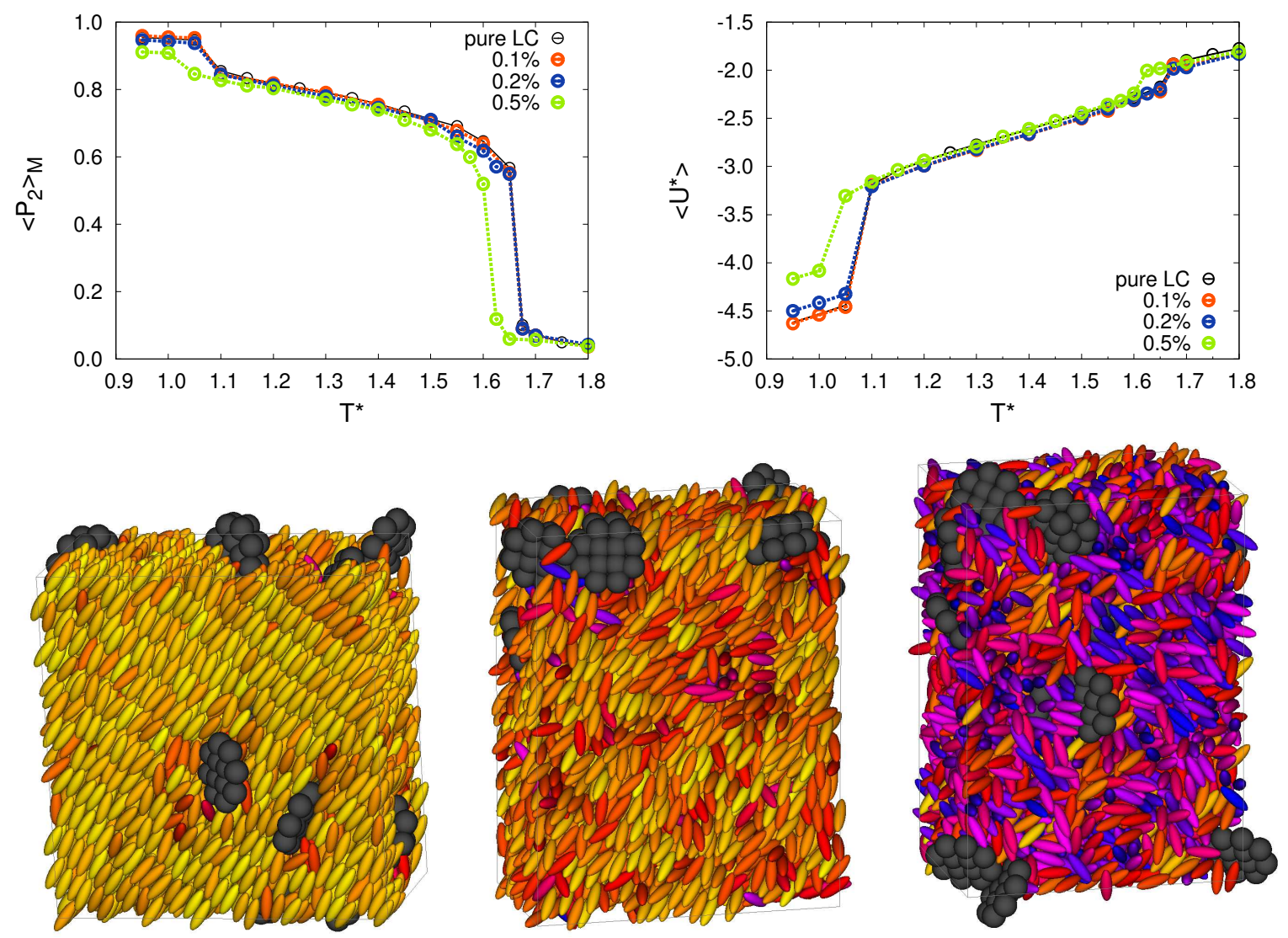

Fig. 13 Average orientational order parameter $\left\langle P_{2}\right\rangle_{M}$ and average dimensionless energy per molecule $\left\langle U^{*}\right\rangle$ against the dimensionless temperature $T^{*}$ for three LC/disc-like NP dispersions, at increasing NP mole fraction $x_{N}$; the data for the pure system are reported here for comparison. Snapshots of $\mathrm{x}_{N}=0.5 \%$ dispersions in solid $\left(T^{*}=1.0\right)$, nematic $\left(T^{*}=1.4\right)$ and Isotropic $\left(T^{*}=1.7\right)$ phase

the low temperature phase. However, as concentration increases, NPs tend to stack face-to-face, thus forming aggregates or just, at our relative dilute concentration dimers. In any case the aggregates being less anisometric (more spherical) than the single particle start to destablize the nematic phase, shifting down $T_{N I}$ (see fig.13).

The contour map of the heterogeneous anisotropic radial correlation function $g\left(r, \cos \beta_{r}\right)_{N M}$ for a $0.1 \%$ sample in the $\mathrm{N}$ phase (Fig.16) shows two broad rings; the first, ranging from $r^{*} \approx$ $1.5-2.0$ to $r^{*} \approx 2.0-2.5$, exhibits the maximum intensity when $\cos \beta_{r}<0.4$ (corresponding to a planar anchoring of the mesogens on the disc face - label $\boldsymbol{P}_{F}$ in the snapshot). The less intense ring, starting from $r^{*} \approx 3-4$, is ascribable to mesogens positioned at the edges of the NP: the anchoring is homeotropic for $\cos \beta_{r}=1$, $r^{*} \approx 3.5$ (mesogens labelled as $\boldsymbol{H}$ ), while planar for $\cos \beta_{r}=0$, $r^{*} \approx 4.5$ (mesogens labelled as $\boldsymbol{P}_{E}$ ). However, one should consider that for these large $r^{*}$, the correlation function samples, not only the first molecular shell surrounding the particles, but also the second and third layers, which means the resulting map is given by a sum of contributions of mesogens in different configurations, which cannot be easily resolved. Overall, the planar mesogens are still predominant with respect to the homeotropic ones.

The temperature dependence of the mesogen mean square displacements against $T^{*}$ (reported for the $x_{N}=0.5 \%$ case in Fig. 15 )

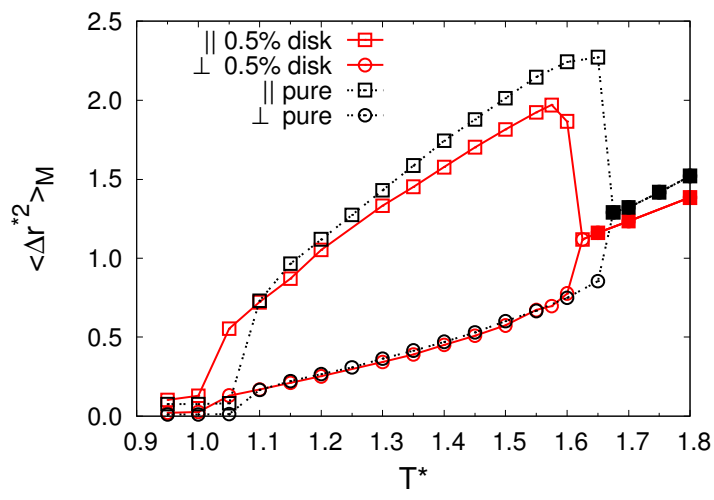

Fig. 15 Temperature dependence of the average MSD components $\left\langle\Delta r_{\|}^{* 2}\right\rangle$ and $\left\langle\Delta r_{\perp}^{* 2}\right\rangle$ measured at $n_{c}=10^{3} \mathrm{MC}$ cycles for the $x_{N}=0.5 \%$ $\mathrm{LC} /$ disc-like NP dispersion (in red). The curves in black report the behaviour of the pure LC system.

shows the same decreasing trend found for the spherical and rodlike NP dispersions, with displacements along the director direction dramatically greater than those perpendicular to it. As for the rod-like NP dispersions, the values of this parallel component are comparable to the ones of the pure GB system in nematic phase, while significatly greater in the low temperature phase. 

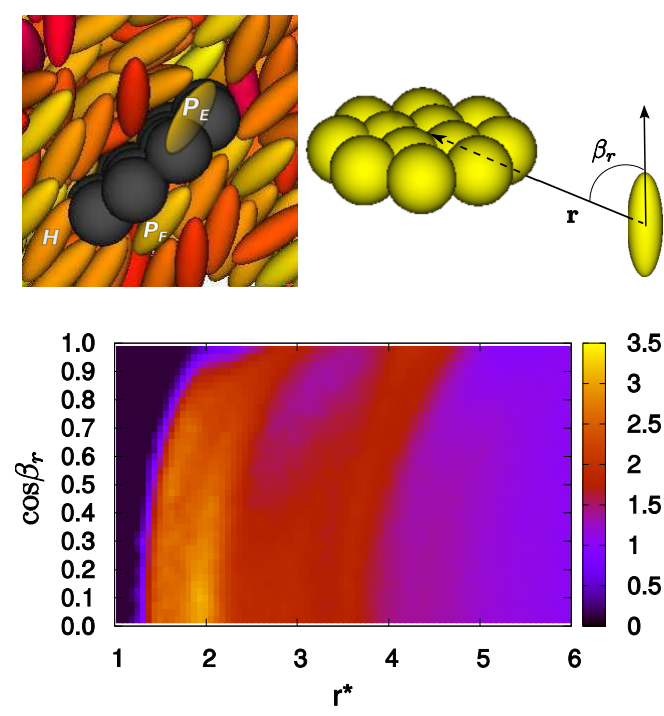

Fig. 16 Longitudinal section of a portion of an instantaneous configuration showing the mesogen anchoring around the disc-shaped and schematic drawing defining the quantities $\mathbf{r}$ and $\beta_{r}$ (top), and contour maps of $g\left(r, \cos \beta_{r}\right)_{N M}$ at $T^{*}=1.4$ for NP fraction $\mathrm{x}_{N}=0.1 \%$ (bottom). The palette on the right indicates the intensity of $g\left(r, \cos \beta_{r}\right)_{N M}$, and ranges from 0 to 3.5 .
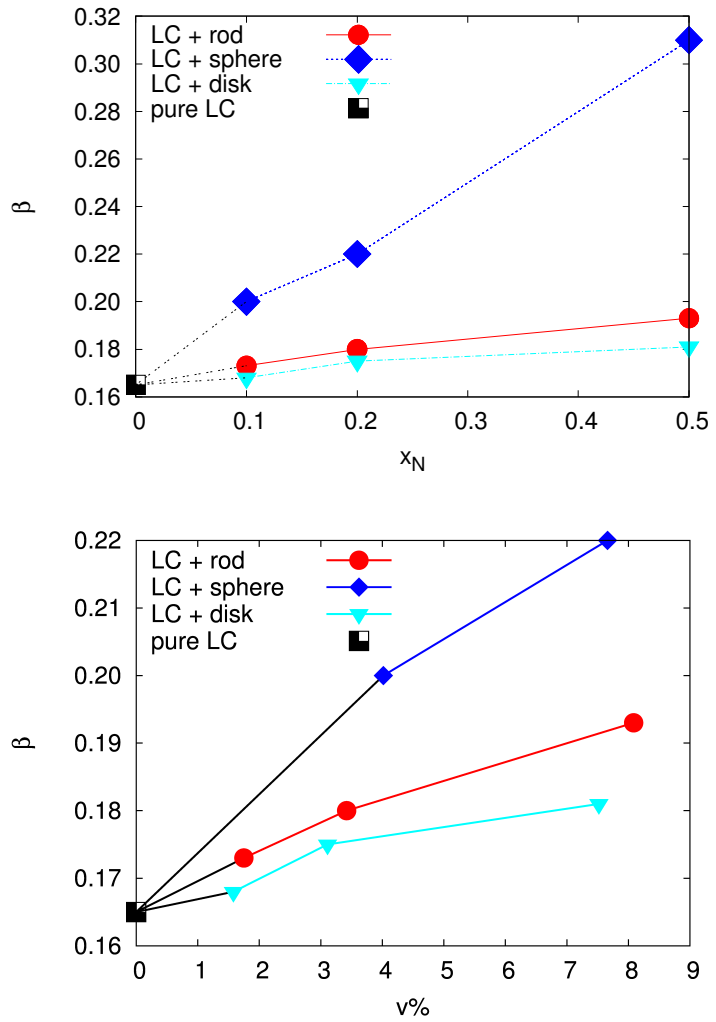

Fig. 17 Plots of the fitted Haller exponents $\beta$ against NP number fraction $x N$ (top) and against the percentage volume fraction of NP, $\mathrm{v} \%$ (bottom) for the three differently shaped NP/LC dispersions.

\section{Discussion and Conclusions}

We have reported the results of Monte Carlo computer simulation studies on dispersions of calamitic mesogens doped with NPs of different shape, but similar largest linear dimension. In the first part of the work we have proposed a novel model to represent NPs of arbitrary shape as suitably arranged tight clusters of rigidly connected spherical units. In particular, we have focused on NPs of overall spherical, rod and disc-like shape and on their effects on the phase behavior, transition temperatures, long-range orientational order and overall structuring of these diluted suspensions at three different NP concentrations: $0.1,0.2,0.5 \%$. These concentrations proved to be low enough to avoid aggregation and phase separation of the NPs.

We have found that for all the NP shapes considered here, the general phase behaviour is not affected by the addition of small amounts $\left(x_{N}=0.1 \%\right)$ of NPs, while a general decrease of the LC orientational order and a change in the trend of the $\left\langle P_{2}\right\rangle_{M}$ vs. $T$ curve is observed in the case of the $x_{N}=0.5 \%$ dispersions, with a more pronounced effect when the NPs are spherical.

The changes of the order parameter dependence calculated for the LC system doped with the three differently-shaped NPs at increasing mole fractions, can be summarized by the behaviour of the Haller exponents $\beta$, shown in Fig. 17 that provide an "at a glance" picture of the extent of the variation of the mesogenic behaviour once the NPs are added, with respect to the pure LC (corresponding to $x_{N}=0$ ). In particular, the spherical NPs system deviates significantly from the pure mesogen behaviour, with a $\beta>0.2$ already at low concentration (namely $0.1 \%$ ), reaching $\beta>0.3$ when NP mole fraction is $0.5 \%$. It is useful to present the data for $\beta$ also as a function of the NP volume fraction, since our NP have different size (Fig. 17, bottom), but the general trends for the different shapes are unchanged, if we compare the interpolated data at the same volume fraction.

Concerning the local structure and the relative mobility of the resulting phases, the addition of an higher fraction $\left(x_{N}=0.5 \%\right)$ of any kind of NPs does not hinder the retention of a certain mobility of the particles, even after entering the low temperature phase, as highlighted by the values of the parallel component of the mean square displacement, and by the oscillations of the $g_{\|}\left(r_{\|}\right)_{M M}$ correlation function which for doped systems never reaches zero, as it would happen in the pure mesogenic system. The contour maps of the anisotropic radial correlation functions and the MC snapshots of the different samples, indicate that the reciprocal arrangement between NP and mesogen is in all cases mainly planar, even though the inhomogeneous alignment leads to the formation of LC orientational defects.

We do not push a comparison with the variety of experimental results available in the literature (see table 1 ) given, as already mentioned, the difficulty of disentangling the various NP features simultaneously present, but we trust our results will be of help in assessing at least the effect of one important NP feature, its shape.

\section{Acknowledgments}

We are grateful for support from USAF - EOARD through grant FA8655-11-1-3046 and from the Air Force Office of Scientific Research. We thank L. Muccioli and M. Ricci for useful discussions. 


\section{References}

1 H. Qi, B. Kinkead and T. Hegmann, Proc. SPIE, 2008, 6911, 691106.

2 H. Qi and T. Hegmann, J. Mat. Chem., 2006, 16, 4197.

3 Y. Shiraishi, N. Toshima, K. Maeds, H. Yoshikawa, J. Xu and S. Kobayashi, Appl. Phys. Lett., 2002, 81, 2845.

4 S. Kobayashi and N. Toshima, Society for Information Display, 2007, 9, 26.

5 H. Yoshida, K. Kawamoto, H. Kubo, T. Tsuda, A. Fujii, S. Kuwabata and M. Ozaki, Adv. Mater., 2010, 22, 622.

6 Y. S. Ha, H. J. Kim, H. G. Park and D. S. Seo, Opt. Exp., 2012, 20, 6448.

7 M. Draper, I. M. Saez, S. J. Cowling, P. Gai, B. Heinrich, B. Donnio, D. Guillon and J. W. Goodby, Adv. Funct. Mater, 2011, 21, 1260.

8 W. Cai and V. Shalaev, "Optical Metamaterials: Fundamentals and Applications", Springer, 2009.

9 M. Mishra, S. Kumar and R. Dhar, RSC Adv., 2014, 4, 62404.

10 P. Kopčanský, N. Tomašovičová, M. Koneracká, M. Timko, Z. Mitróová, V. Závišová, N. Éber, K. Fodor-Csorba, T. TóthKatona, A. Vajda, J. Jadzyn, E. Beaugnon and X. Chaud, Acta Phys. Pol., A, 2010, 33, 988.

11 E. B. Barmatov, D. A. Pedalk and M. V. Barmatova, Liq. Cryst., 2006, 33, 1059.

12 A. Mertelj, L. Cmok, M. Copic, G. Cook and D. R. Evans, Phys. Rev. E, 2012, 85, 021705.

13 S. Krishna Prasad, M. Vijay Kumar, T. Shilpa and C. V. Yelamaggad, RSC Adv., 2014, 4, 4453.

14 V. Anderson and E. Terentjev, Eur. Phys. J. E, 2001, 4, 21-28.

15 N. Tomašovičová, P. Kopčanský, M. Koneracká, L. Tomčo, V. Závišová, M. Timko, N. Éber, K. Fodor-Csorba, T. TóthKatona, A. Vajda and J. Jadzyn, J. Phys.: Condens. Matter, 2008, 20, 204123.

16 M. V. Gorkunov, G. A. Shandryuk, A. M. Shatalova, I. Y. Kutergina, A. S. Merekalov, Y. V. Kudryavtsev, R. V. Talrozeb and M. A. Osipov, Soft Matter, 2013, 9, 3578.

17 H. Qi and T. Hegmann, Liq. Cryst. Today, 2011, 20, 102.

18 S. Kobayashi, Y. Saeki, S. Kodaira, K. Takatoh, T. Kineri, H. Hoshi, N. Toshima and S. Sano, J. Soc. Inform. Display, 2008, 16, 871-874.

19 Y. Reznikov, O. Buchnev, O. Tereshchenko, V. Reshetnyak, A. Glushchenko and J. West, Appl. Phys. Lett., 2003, 82, 1917.

20 A. Arcioni, C. Bacchiocchi, L. Grossi, A. Nicolini and C. Zannoni, J. Phys. Chem. B, 2002, 106, 9245-9251.

21 A. Arcioni, C. Bacchiocchi, I. Vecchi, G. Venditti and C. Zannoni, Chem. Phys. Lett., 2004, 396, 433-441.

22 A. Arcioni, C. Bacchiocchi, I. Vecchi and C. Zannoni, Mol. Cryst. Liq. Cryst., 2005, 429, 213-225.

23 H. Duran, B. Gazdecki, A. Yamashita and T. Kyu, Liq. Cryst., 2005, 32, 815.

24 M. Hakobyan, R. Hakobyan and Y. Chilingaryan, Armen. j. phys., 2014, 7, 11-18.

25 P. Kopčanský, N. Tomašovičová, M. Koneracká, M. Timko,
V. Závišová, A. Džarová, J. Jadzyn, E. Beaugnon and X. Chaud, Int $J$ Thermophys, 2011, 32, 807.

26 F. Li, O. Buchnev, C. Cheon, A. Glushchenko, V. Reshetnyak, Y. Reznikov, T. J. Sluckin and J. L. West, Phys. Rev. Lett., 2006, 97, 147801 .

27 F. Li, O. Buchnev, C. Cheon, A. Glushchenko, V. Reshetnyak, Y. Reznikov, T. J. Sluckin and J. L. West, Phys. Rev. Lett., 2007, 99, 219901.

28 J. Mirzaei, M. Urbanski, H.-S. Kitzerow and T. Hegmann, Phil. Trans. R. Soc. A, 2013, 371, year.

29 M. Kaczmarek, O. Buchnev and I. Nandhakumar, Appl. Phys. Lett., 2008, 92, 103307.

30 Z. Zhang and J. van Duijnevedt, Soft Matter, 2007, 3, 596.

31 O. Kurochkin, H. Atkuri, O. Buchnev, A. Glushchenko, O. Grabar, R. Karapinar, V. Reshetnyak, J. West and Y. Reznikov, Condens. Matter Phys., 2010, 13, 33701.

32 V. Popa-Nita, V. Barna, R. Repnik and S. Kralj, Nanotechnology and Nanomaterials. Syntheses and Applications of Carbon Nanotubes and Their Composites, INTECH, 2013, pp. 145-163.

33 A. Matsuyama, Smart Nanoparticles Technology, INTECH, 2012, pp. 241-268.

34 L. M. Lopatina and J. R. Selinger, Phys. Rev. Lett., 2009, 102, 197802.

35 D. Martire, The Molecular Physics of Liquid Crystals, Academic Press, 1979, pp. 221-238.

36 G. Luckhurst, J. Chem. Soc. Faraday Trans., 1988, 84, 961986.

37 J. Xu, D. Bedrov, G. Smith and M. Glaser, Phys. Rev. E, 2009, 79, 011704.

38 J. Gay and B. Berne, J. Chem. Phys., 1981, 74, 3316.

39 B. Berardi, A. Emerson and C. Zannoni, J. Chem. Soc. Faraday Trans., 1993, 89, 4069.

40 R. Berardi, C. Fava and C. Zannoni, Chem. Phys. Lett., 1998, 297 (1), 8-14.

41 J. G. Gay and B. Berne, J. Chem. Phys., 1981, 74, 3316.

42 C. Zannoni, J. Mat. Chem., 2001, 11, 2637-2646.

43 M. J. Chalam, K. E. Gubbins, E. de Miguel and L. F. Rull, Mol. Simulat., 1991, 7, 357.

44 E. de Miguel, L. F. Rull, M. J. Chalam and K. E. Gubbins, Mol. Phys., 1991, 72, 593.

45 E. de Miguel, L. F. Rull, M. J. Chalam and K. E. Gubbins, Mol. Phys., 1991, 74, 405.

46 E. De Miguel and C. Vega, JCP, 2002, 117, 6313.

47 J. Emsley, G. Luckhurst, W. Palke and D. Tildesley, Liq. Cryst., 1992, 11, 519-530.

48 J. A. Barker and R. O. Watts, Mol. Phys., 1973, 26, 789.

49 C. Zannoni, G. R. Luckhurst and G. W. Gray, The Molecular Physics of Liquid Crystals, Academic Press, 1979, vol. Chap. 3, pp. 51-83.

50 J. T. Brown, M. P. Allen, E. Martín del Rìo and E. de Miguel, Phys. Rev. E, 1998, 57, 6685.

51 I. Haller, PSSC, 1975, 10, 103.

52 R. G. Horn, J. de Physique, 1978, 39, 105. 
53 R. Stannarius, Handbook of Liquid Crystals, Wiley-VCH, 1998, vol. 2A, p. 113.

54 S. T. Wu and R. J. Cox, J. Appl. Phys., 1988, 64, 821-826.

55 I. Chirtoc, M. Chirtoc, C. Glorieux and J. Thoen, Liq. Cryst., 2004, 31, 229-240.

56 G. Tiberio, L. Muccioli, R. Berardi and C. Zannoni, ChemPhysChem, 2009, 10, 125-136.
57 R. Berardi, S. Orlandi and C. Zannoni, Chem. Phys. Lett., 261, 363-368, 1996.

58 M. Palermo, A. Pizzirusso, L. Muccioli and C. Zannoni, J. Chem. Phys., 2013, 138, 204901.

59 Y. Olivier, L. Muccioli and C. Zannoni, ChemPhysChem, 2014, 15, 1345-1355. 